\title{
Phase II trial of SOM230 (pasireotide LAR) in patients with unresectable hepatocellular carcinoma
}

This article was published in the following Dove Press journal: Journal of Hepatocellular Carcinoma

\author{
Lynn G Feun' \\ Medhi Wangpaichitr ${ }^{2}$ \\ Ying-Ying $\mathrm{Li}^{\prime}$ \\ Deukwoo Kwon ${ }^{3}$ \\ Stephen P Richman' \\ Peter J Hosein' \\ Niramol Savaraj ${ }^{1,2}$ \\ 'Department of Medicine, Medical \\ Oncology, Sylvester Comprehensive \\ Cancer Center, University of Miami \\ ${ }^{2}$ Department of Surgery, Miami VA \\ Healthcare System, Research Service, \\ ${ }^{3}$ Biostatistics and Bioinformatics Core, \\ Sylvester Comprehensive Cancer \\ Center, University of Miami, Miami, \\ FL, USA
}

Background: A phase II trial of pasireotide was performed to assess its efficacy and safety in advanced or metastatic hepatocellular carcinoma (HCC).

Patients and methods: Patients with advanced HCC and Child-Pugh score $\leq 7$ received pasireotide LAR $60 \mathrm{mg}$ intramuscularly every 28 days. Primary endpoint was disease control rate. Secondary endpoints were time to tumor progression, response rate, treatment-related adverse events, and overall survival. Serum insulin growth factor-1 was measured before and after pasireotide. Results: Twenty patients were treated and evaluable. Eighteen patients $(90 \%)$ had prior therapy; 16 patients ( $80 \%$ ) had multiple therapies. Median age was $65,75 \%$ had Barcelona Clinic Liver Cancer stage $\mathrm{C}$, and $55 \%$ had metastatic disease. The main toxicity was hyperglycemia. Rare adverse effects included reversible grade 4 elevation in alanina transaminase/aspartate transaminase in one patient. The best response was stable disease in 9 patients (45\%). Median time to tumor progression for the 20 patients was 3 months, and median survival was 9 months.

Conclusion: Pasireotide had limited clinical benefit as second-line or third-line treatment in patients with advanced or metastatic HCC. Low baseline insulin growth factor-1 level may be indicative when SOM230 treatment may be ineffective, and decreasing levels after treatment may be indicative of disease control.

Keywords: pasireotide, hepatocellular carcinoma, insulin growth factor-1

\section{Introduction}

Hepatocellular carcinoma (HCC) is a major health problem worldwide. It is the fifth most common neoplasm in the world and the third most common cause of cancerrelated death. ${ }^{1}$ More than 500,000 new cases are currently diagnosed yearly, with an age-adjusted worldwide incidence of 5.5-14.9 per 100,000 population.

Unfortunately, most HCC patients are diagnosed at an advanced stage and are not candidates for curative therapy. Until recently, there was no standard palliative therapy for patients with advanced or unresectable HCC. In 2007, the results of the SHARP trial ${ }^{2}$ were reported, and this led to the approval of sorafenib (Nexavar, Bayer-Onyx) as the first and only therapeutic agent definitively proven to prolong survival in patients with advanced HCC. Despite having a very low response rate of $2 \%$, sorafenib improved overall survival (OS) and time to radiological progression by 3 months compared to placebo. More recently, regorafenib was shown in a phase III trial to be superior to placebo in sorafenib failure patients. ${ }^{3}$ The OS for regorafenib was 10.6 months compared to 7.8 months for placebo. Time to tumor progression was 3.2 months for regorafenib and 1.5 months for placebo. Disease control rate and response rate was
Correspondence: Lynn G Feun Department of Medicine, Medical Oncology, Sylvester Comprehensive Cancer Center, University of Miami, 1475 NW 12th Avenue, Miami, FL 33136, USA

Tel +l 3052436606

$\mathrm{Fax}+\mathrm{I} 3052434975$

Email Ifeun@med.miami.edu 
$65.2 \%$ and $10.6 \%$ for regorafenib and $36.1 \%$ and $4.1 \%$ for placebo, respectively. These results show that regorafenib had modest clinical benefit as OS and time to progression (TTP) were still $<3$ months over that of placebo.

Most patients with HCC have coexistent cirrhosis with portal hypertension which can predispose to therapy-related leukopenia and thrombocytopenia. Therefore, the ideal anticancer drug for advanced HCC would not require hepatic metabolism, and would have minimal or no bone marrow suppression. The somatostatin analog octreotide is an agent which fulfills these requirements. Octreotide is effective in the treatment of neuroendocrine tumors, principally by reducing symptomatic hormonal secretion, but also by a direct antineoplastic effect. ${ }^{4,5}$ These results led to a wider application of octreotide in the treatment of other solid malignancies, including in advanced HCC. ${ }^{6,7}$

The molecular mechanisms involved in the antineoplastic activity of somatostatin relate to direct and indirect growth inhibitory effects mediated by specific somatostatin receptors (SSTRs) expressed on target tissues. ${ }^{4,8}$ Verhoef et $\mathrm{al}^{9}$ recently reported a detection rate of $67 \%$ for the SSTR-2 in human HCC tissues. Activation of SSTRs directly inhibits cell proliferation and induced cell death by apoptosis. ${ }^{4}$ Indirect antineoplastic effects include reduced or inhibited secretion of growth-promoting hormones and growth factors, and the inhibition of angiogenesis. ${ }^{4,8}$ Approximately $40 \%$ of advanced HCC express SSTRs, ${ }^{10}$ and in vitro data suggest a direct antitumor effect of octreotide in advanced HCC. ${ }^{11}$ Taking these data together with the superior safety profile of octreotide and its favorable results in reducing portal hypertension, ${ }^{12}$ there exists a good rationale for the evaluation of somatostatin analogs like octreotide in advanced HCC.

Like natural somatostatin and other somatostatin analogs, pasireotide exerts its pharmacological activity via binding to SSTR's. There are five known SSTRs: SSTR-1, 2, 3, 4, and 5. SSTRs are expressed in different tissues under normal physiological conditions. Somatostatin analogs activate these receptors with different potencies, ${ }^{13}$ and this activation results in a reduced cellular activity and inhibition of hormone secretion. The somatostatin analogs currently approved for use in the clinic (octreotide and lanreotide) have a high affinity to the SSTR-2, with moderate or no affinity to the remaining subtypes. Pasireotide is a novel cyclohexapeptide somatostatin analog that exhibits a unique binding profile, binding with high affinity to 4 of the 5 known human SSTRs. Compared to Sandostatin $^{\circledR}$ (octreotide acetate), pasireotide exhibits a binding affinity, which is 30-40 times higher for human SSTR-1 and SSTR-5, 5 times higher for human SSTR-3, and 2.5 times lower for human SSTR-2. There are also in vivo data using a HCC xenograft model showing efficacy of pasireotide. ${ }^{14}$

Thus, there is a strong rationale to evaluate pasireotide in a phase II trial in advanced HCC. We report here the first in-human clinical phase II trial of single-agent pasireotide in this disease. In addition to the therapeutic trial, correlative laboratory study was performed with insulin growth factor (IGF) blood measurements before and during treatment. ${ }^{15}$

\section{Patients and methods}

The study was approved by University of Miami institutional review board and registered with clinicaltrials.gov (NCT01639352). All patients signed written informed consent for this study.

This was a phase II trial for patients who had been diagnosed with advanced unresectable or metastatic HCC.

Eligibility criteria included patients 18 years of age or older with a diagnosis of HCC (either biopsy proven or with radiographic evidence consistent with $\mathrm{HCC}$ and elevated serum alpha fetoprotein $>400 \mathrm{ng} / \mathrm{mL}$ or findings on magnetic resonance imaging or computed tomography scans characteristic of a primary liver tumor and cirrhosis). All patients had to have been off prior therapy for at least 4 weeks and had actively progressing disease.

Other criteria include Karnofsky performance status of 80 or Eastern Cooperative Oncology Group performance status of 0 or 1 , life expectancy $\geq 12$ weeks, adequate bone marrow function as shown by: absolute neutrophil count $\geq 1.2 \times 10^{9} / \mathrm{L}$, platelets $\geq 50 \times 10^{9} / \mathrm{L}$, adequate liver function defined as serum bilirubin $<1.5 \times U L N$ and serum transaminases activity $\leq 3 \times$ $\mathrm{ULN}$, serum prothrombin time $\leq 16$ seconds, serum creatinine $\leq 1.5 \times \mathrm{ULN}$, fasting serum cholesterol $\leq 300 \mathrm{mg} / \mathrm{dL} \mathrm{OR} \leq 7.5$ $\mathrm{mmol} / \mathrm{L}$ and fasting triglycerides $\leq 2.5 \times \mathrm{ULN}$, Child-Pugh A and early Child's B (no more than 7 points). Patients were excluded if they had received prior octreotide therapy or any somatostatin analog. Other exclusions included the following: presence of uncontrolled diabetes mellitus (defined as HbA1c $>7 \%$ or $=8 \%$ despite therapy, or fasting plasma glucose $>1.5$ ULN, symptomatic cholelithiasis, baseline QTcF $>470 \mathrm{~ms}$. QTcF is Fridericia's correction formula takes into account the physiologic shortening of the QT interval which occurs as the heart rate increases, permitting comparison of the QT interval across a range of rates. It is mathematically defined as $\mathrm{QTcF}=\mathrm{QT} / \mathrm{CubeRoot} \mathrm{RR}$ (seconds).

Each patient enrolled in the study received long-acting pasireotide administered intramuscularly (IM) intragluteally. The starting dose for all patients was $60 \mathrm{mg}$ IM every 28 days. There was no dose escalation beyond $60 \mathrm{mg}$ every 28 days. 
Electrocardiography (EKG) monitoring included baseline before 1 st dose, 21 days after 1st dose, 21 days after 3rd dose, and prior to injection on cycle 6 . Subsequently, EKG was performed every 3 cycles after cycle 6 (prior to drug injection). Magnetic resonance imaging or computed tomography scans of the abdomen were done at start of treatment and then every 8 weeks. Treatment response was determined by RECIST 1.1 criteria. For correlative study, serum for biomarker IGF-1 was performed at baseline, day 29, and day 57 and assayed with an enzyme-linked immunosorbent assay using the kit from R\&D (Minneapolis, MN, USA). All sample analyses were done in duplicate.

For statistical analysis, Kaplan-Meier estimate was performed for time to tumor progression and OS.

\section{Results}

Twenty-six patients were enrolled for the study. Twenty of these patients were considered eligible and were treated (6 patients failed screening, mainly due to elevated liver function tests). The first patient was enrolled in July 2012. In terms of patient characteristics, the median age was 65 (range 52-81), with most patients being of male sex, and hepatitis $\mathrm{C}$ infection was the most common etiology. The baseline characteristics are shown in Table 1. The majority (14 patients) had prior sorafenib and 6 patients refused sorafenib (6 patients). Only 2 patients had no prior therapy, while 16 patients had multiple prior therapies, including transarterial chemoembolization in 14 patients and Ytrium 90 in 2 patients. The median Karnofsky performance status

Table I Baseline characteristics of 20 treated patients

\begin{tabular}{ll}
\hline Characteristic & N (\%) \\
\hline Age at diagnosis (years) & Median 65 (range 52-8I) \\
Male sex & $15(75)$ \\
Child-Pugh class A & $19(95)$ \\
BCLC stage & \\
B & $5(25)$ \\
C & $15(75)$ \\
Etiology & \\
Hepatitis B & $3(15)$ \\
Hepatitis C & $8(40)$ \\
Hepatitis B and C & $2(10)$ \\
Prior treatments & \\
None & $2(10)$ \\
TACE & $12(60)$ \\
Chemotherapy & $2(10)$ \\
Ablation & $6(30)$ \\
Yttrium 90 & $2(10)$ \\
Cryoablation & $1(5)$ \\
Sorafenib & $14(70)$ \\
\hline
\end{tabular}

Note: apatients may have had multiple therapies.

Abbreviations: BCLC, Barcelona Clinic Liver Cancer stage C; TACE, transarterial chemoembolization. was 90 (range 100-90), and Eastern Cooperative Oncology Group performance status median was 1 (range 0-1). Fifteen patients (75\%) had Barcelona Clinic Liver Cancer stage $\mathrm{C}$ stage C, 5 had Barcelona Clinic Liver Cancer stage C stage $\mathrm{B}$, and 11 patients $(55 \%)$ had metastatic disease.

In terms of safety, the main toxicity noted was hyperglycemia, particularly in patients with history of diabetes mellitus (Table 2). Toxicities included 17 hyperglycemia episodes in 8 patients. One grade 4 hyperglycemia episode occurred in a diabetic patient who neglected to take his morning insulin (not likely drug related). Other hyperglycemia episodes include 6 grade 3 episodes (4 probable related and 2 possible), 8 grade 2 episodes ( 4 probable and 4 possible), and 2 grade 1 (1 definite, 1 possible) episodes. No patient discontinued therapy due to hyperglycemia. Other adverse effects were uncommon. One patient had grade 4 reversible elevation of liver function tests (alanine transaminase/aspartate transaminase), which returned to grade 1 within 3 weeks. CT scan showed progressive liver cancer, and so the patient was taken off study. Three patients had fatigue, one patient had gastrointestinal bleeding (considered to be unrelated to study drug), and one patient had diarrhea (grade 1). No prolonged QTcF in the EKG was noted in any patient requiring drug delay or withdrawal.

In terms of antitumor response and efficacy, no patient had a complete or partial response. Nine (45\%) of the 20 patients

Table 2 Frequency of Grade 3-4 adverse events

\begin{tabular}{lll}
\hline Adverse event & Grade 3, N (\%) & Grade 4, N (\%) \\
\hline Hyperglycemia & $6(30)$ & I (5) \\
AST or ALT elevation & $5(25)$ & $3(15)$ \\
Hypoglycemia & I (5) & I (5) \\
Hypercalcemia & I (5) & I (5) \\
Mucositis & I (5) & \\
Creatinine elevation & I (5) & I (5) \\
INR elevation & I (5) & \\
Vomiting & & I (5) \\
Upper GI bleeding & & I (5) \\
Anal bleeding & & I (5) \\
Hepatic infection & & I (5) \\
Hyponatremia & & I (5) \\
Liver failure & & I (5) \\
Respiratory failure & & I (5) \\
Anemia & & I (5) \\
Fatigue & I (5) \\
Hepatic encephalopathy & I (5) \\
Lung infection & $\mathrm{I}(5)$ & \\
Bilirubin elevation & $\mathrm{I}(5)$ & \\
Gallbladder obstruction & $\mathrm{I}(5)$ & \\
Abdominal pain & $\mathrm{I}(5)$ & \\
Alkaline phosphatase elevation & $\mathrm{I}(5)$ & \\
\hline Abbreviations: ALT, &
\end{tabular}

Abbreviations: ALT, alanine transaminase; AST, aspartate transaminase; INR, international normalized ratio; Gl, gastrointestinal. 
had minor response/stable disease after 2 cycles; of these, $5(25 \%)$ had continued minor response/stable disease after 4 cycles. The median TTP for the 20 patients was 3 months (95\% confidence interval for median TTP $=[2,4]$, Figure 1$)$. The median OS was 9 months (95\% confidence interval for median OS $=[4, \mathrm{NA}]$, Figure 2). Seven patients were alive

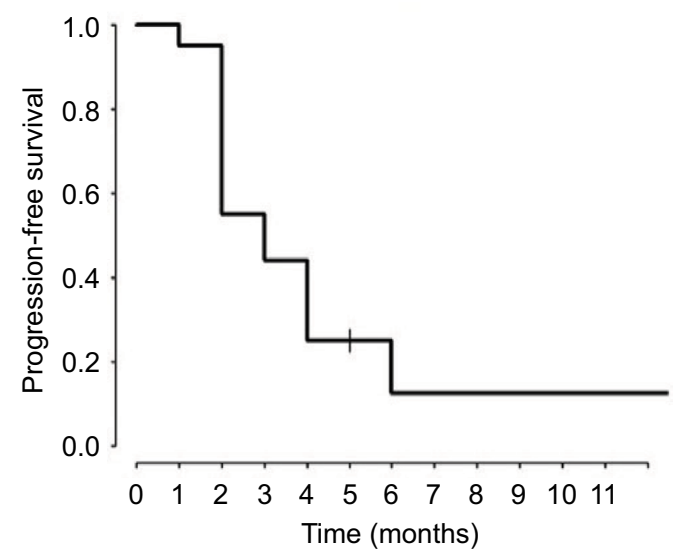

Figure I Kaplan-Meier plot of estimate TTP. Abbreviation: TTP, time to progression.

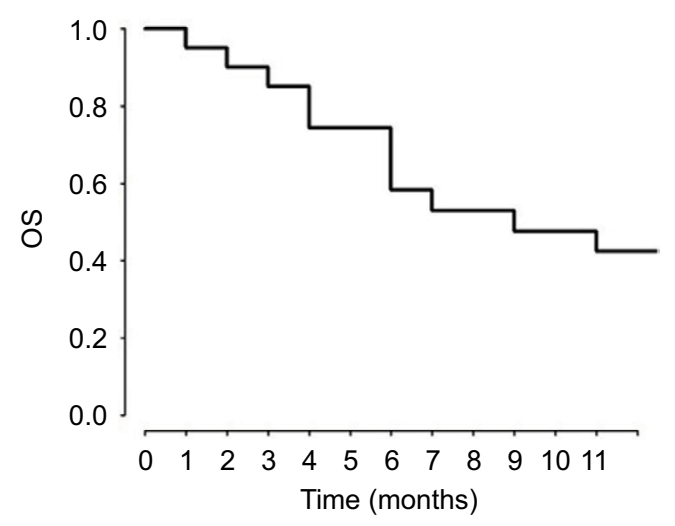

Figure 2 Kaplan-Meier plot of estimate of OS.

Abbreviation: OS, overall survival.
$(3+, 17+, 20+, 23+, 24+, 30+$, and $54+$ months $)$ at the discontinuation of the study.

For the correlative laboratory study, serum IGF-1 was measured in patients before and after pasireotide. Baseline levels varied from 15 to $129 \mathrm{ng} / \mathrm{mL}$ (average $48.763 \mathrm{ng} / \mathrm{mL}$ ). Decreasing serum IGF-1 after pasireotide treatment by 2.5-16 fold occurred in all patients who had minor response/ stable disease (Figure 3). In one patient with progressive disease, no change in IGF levels was detected. However, levels start to rise with disease progression. While the number of patients are limited, patients who had baseline IGF-1 levels $<20 \mathrm{ng} / \mathrm{mL}$ had a shorter median TTP of 1.5 months, while patients who had IGF-1 levels $>20 \mathrm{ng} / \mathrm{mL}$ had a median TTP of 5 months. TTP was compared between the two group using the log-rank test with the $p$-value of 0.0075 which is significant. Overall, our data suggest that low baseline IGF-1 level is indicative when pasireotide treatment may be ineffective, and decreasing IGF-1 levels after treatment may be indicative of disease control.

\section{Discussion}

Currently, there is no approved drug in the US for second therapy after sorafenib failure or progression. More recently, regorafenib has shown superiority over placebo in both time to tumor progression and OS. ${ }^{3}$ Toxicity from regorafenib included grade 3-4 hypertension 15\%, hand-foot skin reaction $13 \%$, fatigue $9 \%$, and diarrhea $3 \%$. Serious adverse events occurred in $44 \%$ of regorafenib patients. Also, $25 \%$ of patients in the regorafenib group discontinued treatment due to adverse events. The most common adverse events which resulted in discontinuation of regorafenib over placebo were increase in alanine transaminase, hand-foot skin reaction, and increase in alanine transaminase. Thus, while regorafenib is the first drug in randomized trials to show benefit over
A

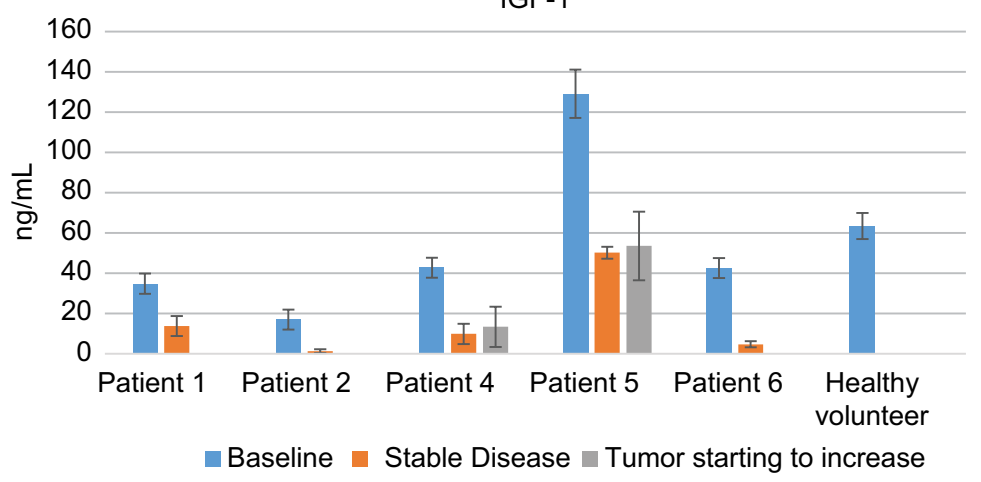

B

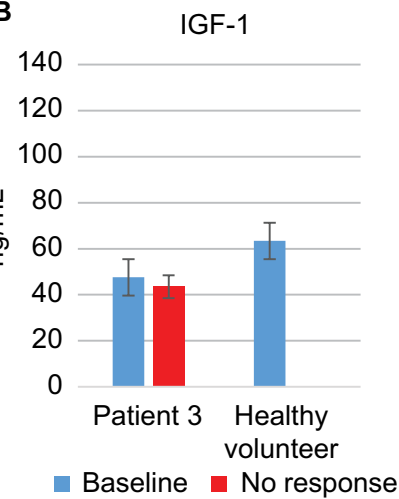

Figure 3 IGF-I concentration.

Notes: (A) Patients at baseline (blue), at time of minor response-stable disease (orange) and at time tumor starting to increase (gray). (B) Patients at baseline, patient with no response (red).

Abbreviation: IGF-I, insulin growth factor-I. 
placebo in second-line therapy for HCC, a quarter of the patients had to discontinue therapy due to adverse events. Other drugs in randomized phase III trials have not demonstrated statistical benefit over placebo. ${ }^{16-18}$

Somatostatin analogs including pasireotide may have a role due to low toxicity and possible antitumor effect. Previous studies have suggested some benefit for HCC patients. There are now numerous reports in the literature exploring the activity of somatostatin analogs in advanced HCC. There are at least three reported cases of prolonged complete remissions of HCC with octreotide therapy. ${ }^{19-21}$ Unfortunately, prospective trials assessing the efficacy of somatostatin analogs in HCC have been conflicting. In some studies, octreotide was found to significantly improve OS of patients with unresectable $\mathrm{HCC},{ }^{22-25}$ including three randomized controlled trials, while these results were not confirmed by other studies, ${ }^{26-29}$ including three randomized controlled trials. A recent metaanalysis concluded that there was no net OS benefit in HCC patients treated with octreotide. ${ }^{30}$ Despite the inconsistent results seen with trials of octreotide, there remains a subset of HCC patients who derive benefit from somatostatin analogs. The heterogeneity of the data suggests that patient selection is the key to maximizing the therapeutic potential of this class of drugs.

A candidate biomarker which may predict for benefit is SSTR expression, which can be noninvasively assessed by radiolabeled octreotide scintigraphy. Interestingly, only one of the randomized trials discussed above selected patients a priori for positive SSTR expression by means of ${ }^{111}$ Indium octreotide scintigraphy. ${ }^{24}$ In this study, 127 patients were enrolled, and 66 (52\%) had negative octreoscans. Among the 61 patients with positive octreoscans, 31 were randomized to octreotide and 30 to placebo. The OS was significantly better in SSTR-positive patients treated with octreotide vs placebo (49 vs 28 weeks, $p<0.001$ and compared to 28 weeks for the SSTR negative group). Another study by the same authors had shown a significant difference in the median survival time ( 31 vs 16 weeks, $p=0.037$ ) and an improvement in the quality of life (60\% vs $23 \%$ ) in 28 cirrhotic patients with HCC using the octreoscan for detection of SSTR. Only patients with intense uptake in the liver on octreoscan were treated with long-acting octreotide. ${ }^{23}$ The survival benefit seen in these two well-designed trials provides the impetus to study pasireotide, whose binding affinity for SSTR-1, 3, and 5 is significantly higher compared to octreotide. There is evidence that multiple SSTR subtypes are expressed in hepatomas, ${ }^{31}$ and the higher affinity of pasireotide to these subtypes may confer a therapeutic advantage.
In this pretreated patient population with advanced, unresectable HCC, pasireotide given as second-line or third-line therapy showed modest benefit in disease control and OS. This is the first in-human study to evaluate singleagent pasireotide in HCC. Recently, a phase II trial of the combination of everolimus and pasireotide in $\mathrm{HCC}$ was reported..$^{32}$ This study was terminated following results that showed no benefit with everolimus in HCC. ${ }^{33}$ This prompted an unplanned interim analysis that found the conditional probability of rejecting the null hypothesis based on events in the 24 patients treated was 0.08 . The results of the combination of everolimus and pasireotide showed a median TTP of 3.5 months and median survival of 6.7 months. The best response was stable disease in 10 patients. Adverse events of the combination therapy were grade 3 hyperglycemia in 6 patients $(25 \%)$. There were no grade 4 toxicity events. The TTP in our study was similar (3 months), while the superior median OS of 9 months in our study may reflect patient selection, post pasireotide therapy, etc.

Although the results in our trial showed very modest disease control in advanced, unresectable HCC as a second- or third-line treatment, future trials may select patients most likely to benefit, such as using pretreatment octreotide image scanning and testing available tumor samples for SSTR expression. Unfortunately, due to costs of octreotide scanning and insurance issues, we were not able to select out the best patients to benefit from this therapy. Baseline serum IGF-1 levels and decreasing IGF-1 after treatment may have value in assessing tumor control or possibly predict better clinical outcome. Further studies will be needed to confirm its utility in this setting.

More recently, immunotherapy has shown promise in HCC. ${ }^{34,35}$ Checkpoint inhibitors such as tremelimumab and nivolumab have shown activity in patients with advanced HCC. ${ }^{36,37}$ Both octreotide and pasireotide have antiproliferative effect on human lymphocytes ${ }^{38}$ and may alleviate the diarrhea from checkpoint inhibitor treatment. Whether somatostatin analogs would enhance or inhibit immune effects of these drugs is not known and will need further study.

\section{Conclusion}

Pasireotide as single agent has limited and very modest clinical benefit as second-line or third-line therapy for HCC. Recent and ongoing trials with FGFR inhibitor or MET inhibitor ${ }^{39}$ have shown promise in HCC. Combining with other targeted agents such as FGFR inhibitor, MET, or MEK inhibitor ${ }^{40}$ is possible due to its low toxicity profile of pasireotide. 


\section{Acknowledgment}

This work was supported by a grant from Novartis.

\section{Author contributions}

Conception and design of the study was done by L G Feun and N Savaraj. Administrative support was provided by all the authors. Study material provision or patient selection was done by L G Feun, S P Richman, P J Hosein, and N Savaraj. Data were collected and assembled by L G Feun, M Wangpaichitr, and N Savaraj. Data analysis and interpretation was done by $\mathrm{L}$ G Feun, M Wangpaichitr, Y-Y Li, D Kwon, and N Savaraj. L G Feun and N Savaraj wrote the manuscript. All authors contributed toward data analysis, drafting and critically revising the paper and agree to be accountable for all aspects of the work.

\section{Disclosure}

The authors report no conflicts of interest in this work.

\section{References}

1. Parkin DM, Bray F, Ferlay J, Pisani P. Estimating the world cancer burden: Globocan 2000. Int J Cancer. 2001;94(2):153-156.

2. Llovet JM, Ricci S, Mazzaferro V, et al. Sorafenib in advanced hepatocellular carcinoma. $N$ Engl J Med. 2008;359(4):378-390.

3. Bruix J, Qin S, Merle P, et al. Regorafenib for patients with hepatocellular carcinoma who progressed on sorafenib treatment (RESORCE): a randomised, double-blind, placebo-controlled, phase 3 trial. Lancet. 2017;389(10064):56-66.

4. Lamberts SW, van der Lely AJ, de Herder WW, Hofland LJ. Octreotide. N Engl J Med. 1996;334(4):246-254.

5. Shojamanesh H, Gibril F, Louie A, et al. Prospective study of the antitumor efficacy of long-term octreotide treatment in patients with progressive metastatic gastrinoma. Cancer. 2002;94(2):331-343.

6. Scarpignato C, Pelosini I. Somatostatin analogs for cancer treatment and diagnosis: an overview. Chemotherapy. 2001;47(Suppl 2):1-29.

7. Hejna M, Schmidinger M, Raderer M. The clinical role of somatostatin analogues as antineoplastic agents: much ado about nothing? Ann Oncol. 2002;13(5):653-668.

8. Froidevaux S, Eberle AN. Somatostatin analogs and radiopeptides in cancer therapy. Biopolymers. 2002;66(3):161-183.

9. Verhoef C, van Dekken H, Hofland LJ, et al. Somatostatin receptor in human hepatocellular carcinomas: biological, patient and tumor characteristics. Dig Surg. 2008;25(1):21-26.

10. Reubi JC, Zimmermann A, Jonas S, et al. Regulatory peptide receptors in human hepatocellular carcinomas. Gut. 1999;45(5):766-774.

11. Papalampros E, Felekouras ES, Filis K, et al. Liver pathology and cell proliferation after octreotide administration following partial hepatectomy in rats: an experimental study. Dig Dis Sci. 2002;47(9):1953-1958.

12. Burroughs AK, Panagou E. Pharmacological therapy for portal hypertension: rationale and results. Semin Gastrointest Dis. 1995;6(3):148-164.

13. Schmid HA, Schoeffter P. Functional activity of the multiligand analog SOM230 at human recombinant somatostatin receptor subtypes supports its usefulness in neuroendocrine tumors. Neuroendocrinology. 2004;80(Suppl 1):47-50.

14. Xie Y, Chen S, Wang CH, Tang CW. Induction of necrosis in the hepatocellular carcinoma HepG2 xenografts treated with SOM230. Zhonghua Gan Zang Bing Za Zhi. 2009;17(10):759-764.

15. Kaseb AO, Morris JS, Hassan MM, et al. Clinical and prognostic implications of plasma insulin-like growth factor-1 and vascular endothelial growth factor in patients with hepatocellular carcinoma. J Clin Oncol. 2011;29(29):3892-3899.
16. Llovet JM, Decaens T, Raoul JL, et al. Brivanib in patients with advanced hepatocellular carcinoma who were intolerant to sorafenib or for whom sorafenib failed: results from the randomized phase III BRISK-PS study. J Clin Oncol. 2013;31(28):3509-3516.

17. Cainap C, Qin S, Huang WT, et al. Linifanib versus sorafenib in patients with advanced hepatocellular carcinoma: results of a randomized phase III trial. J Clin Oncol. 2015;33(2):172-179.

18. Zhu AX, Park JO, Ryoo BY, et al. Ramucirumab versus placebo as second-line treatment in patients with advanced hepatocellular carcinoma following first-line therapy with sorafenib $(\mathrm{REACH})$ : a randomised, double-blind, multicentre, phase 3 trial. Lancet Oncol. 2015;16(7):859-870.

19. Deming DA, Stella AL, Holen KD, Ku G, O’Reilly EM. A dramatic response to long-acting octreotide in metastatic hepatocellular carcinoma. Clin Adv Hematol Oncol. 2005;3(6):468-472; discussion 472-464.

20. Siveke JT, Herberhold C, Folwaczny C. Complete regression of advanced HCC with long acting octreotide. Gut. 2003;52(10):1531.

21. Rahmi G, Malka D, Tomasic G, Dromain C, Ducreux M, Boige V. Complete, long-standing regression of hepatocellular carcinoma after somatostatin analogue treatment. J Clin Oncol. 2007;25(16):e15-e16.

22. Kouroumalis E, Skordilis P, Thermos K, Vasilaki A, Moschandrea J, Manousos ON. Treatment of hepatocellular carcinoma with octreotide: a randomised controlled study. Gut. 1998;42(3):442-447.

23. Dimitroulopoulos D, Xinopoulos D, Tsamakidis K, et al. The role of sandostatin LAR in treating patients with advanced hepatocellular cancer. Hepatogastroenterology. 2002;49(47):1245-1250.

24. Dimitroulopoulos D, Xinopoulos D, Tsamakidis K, et al. Long acting octreotide in the treatment of advanced hepatocellular cancer and overexpression of somatostatin receptors: randomized placebo-controlled trial. World J Gastroenterol. 2007;13(23):3164-3170.

25. Samonakis DN, Moschandreas J, Arnaoutis T, et al. Treatment of hepatocellular carcinoma with long acting somatostatin analogues. Oncol Rep. 2002;9(4):903-907.

26. Yuen MF, Poon RT, Lai CL, et al. A randomized placebo-controlled study of long-acting octreotide for the treatment of advanced hepatocellular carcinoma. Hepatology. 2002;36(3):687-691.

27. Becker G, Allgaier HP, Olschewski M, Zahringer A, Blum HE, Group HS. Long-acting octreotide versus placebo for treatment of advanced HCC: a randomized controlled double-blind study. Hepatology. 2007;45(1):9-15.

28. Slijkhuis WA, Stadheim L, Hassoun ZM, et al. Octreotide therapy for advanced hepatocellular carcinoma. J Clin Gastroenterol. 2005;39(4): 333-338.

29. Barbare JC, Bouche O, Bonnetain F, et al. Treatment of advanced hepatocellular carcinoma with long-acting octreotide: a phase III multicentre, randomised, double blind placebo-controlled study. Eur $J$ Cancer. 2009;45(10):1788-1797.

30. Guo TK, Hao XY, Ma B, et al. Octreotide for advanced hepatocellular carcinoma: a meta-analysis of randomized controlled trials. J Cancer Res Clin Oncol. 2009;135(12):1685-1692.

31. Blaker M, Schmitz M, Gocht A, et al. Differential expression of somatostatin receptor subtypes in hepatocellular carcinomas. J Hepatol. 2004;41(1):112-118.

32. Sanoff HK, Kim R, Ivanova A, Alistar A, McRee AJ, O’Neil BH. Everolimus and pasireotide for advanced and metastatic hepatocellular carcinoma. Invest New Drugs. 2015;33(2):505-509.

33. Zhu AX, Kudo M, Assenat E, et al. Effect of everolimus on survival in advanced hepatocellular carcinoma after failure of sorafenib: the EVOLVE-1 randomized clinical trial. JAMA. 2014;312(1):57-67.

34. Heo J, Reid T, Ruo L, et al. Randomized dose-finding clinical trial of oncolytic immunotherapeutic vaccinia JX-594 in liver cancer. Nat Med. 2013;19(3):329-336.

35. El Ansary M, Mogawer S, Elhamid SA, et al. Immunotherapy by autologous dendritic cell vaccine in patients with advanced HCC. $J$ Cancer Res Clin Oncol. 2013;139(1):39-48.

36. Sangro B, Gomez-Martin C, de la Mata M, et al. A clinical trial of CTLA-4 blockade with tremelimumab in patients with hepatocellular carcinoma and chronic hepatitis C. J Hepatol. 2013;59(1):81-88. 
37. El-Khoueiry AB, Melero I, Crocenzi TS, et al. Phase I/II safety and antitumor activity of nivolumab in patients with advanced hepatocellular carcinoma (HCC): CA209-040. J Clin Oncol. 2015;33(Suppl): abstr LBA101.

38. Lattuada D, Casnici C, Crotta $\mathrm{K}$, et al. Inhibitory effect of pasireotide and octreotide on lymphocyte activation. J Neuroimmunol. 2007; 182(1-2):153-159.
39. Santoro A, Rimassa L, Borbath I, et al. Tivantinib for second-line treatment of advanced hepatocellular carcinoma: a randomized, placebo controlled phase 2 study. Lancet Oncol. 2013;14(1):555-563.

40. Facciorusso A, Licinio R, Carr BI, Di Leo A, Barone M. MEK 1/ 2 inhibitors in the treatment of hepatocellular carcinoma. Expert Rev Gastroenterol Hepatol. 2015;9(7):993-1003.

\section{Publish your work in this journal}

The Journal of Hepatocellular Carcinoma is an international, peerreviewed, open access journal that offers a platform for the dissemination and study of clinical, translational and basic research findings in this rapidly developing field. Development in areas including, but not limited to, epidemiology, vaccination, hepatitis therapy, pathology and molecular tumor classification and prognostication are all considered for publication. The manuscript management system is completely online and includes a very quick and fair peer-review system, which is all easy to use. Visit http://www.dovepress.com/testimonials.php to read real quotes from published authors.

Submit your manuscript here: https:/www.dovepress.com/journal-of-hepatocellular-carcinoma-journal 